\title{
Deep convolutional neural networks for estimating porous material parameters with ultrasound tomography
}

\author{
Timo Lähivaara $^{a}$, Leo Kärkkäinen ${ }^{b}$, Janne M.J. Huttunen $^{b}$, and Jan S. Hesthaven ${ }^{c}$ \\ ${ }^{a}$ Department of Applied Physics, University of Eastern Finland, Kuopio, Finland \\ ${ }^{b}$ Digital Health Laboratory, Nokia Technologies, Espoo, Finland \\ ${ }^{c}$ Computational Mathematics and Simulation Science, Ecole Polytechnique Fédérale de \\ Lausanne, Lausanne, Switzerland
}

\begin{abstract}
We study the feasibility of data based machine learning applied to ultrasound tomography to estimate watersaturated porous material parameters. In this work, the data to train the neural networks is simulated by solving wave propagation in coupled poroviscoelastic-viscoelastic-acoustic media. As the forward model, we consider a high-order discontinuous Galerkin method while deep convolutional neural networks are used to solve the parameter estimation problem. In the numerical experiment, we estimate the material porosity and tortuosity while the remaining parameters which are of less interest are successfully marginalized in the neural networks-based inversion. Computational examples confirms the feasibility and accuracy of this approach.
\end{abstract}

\section{INTRODUCTION}

The porosity of a material is the ratio of the non-solid matter (gas or liquid) to the total volume of media. Information on the porosity of the media is required in many applications. Porosity defines, for example, how much water can be stored in soil or bedrock (groundwater and soil structure studies), how strong human bones are (osteoporosis diagnostics), what the thermal insulating ability of a material is (structural engineering) or how a material absorbs sound (building acoustics).

Transport of gas or liquid in the porous medium affects the bonding of the pores of the material. Physically, this property is measured by flow resistivity and tortuosity. The flow resistivity defines how the porous material resists the flow of gas or liquid through it. Tortuosity describes the complexity of porous "channels" of the material. The porous material may be good at storing the liquid but release it very slowly (e.g. clay) or the material may exhibit high porosity and low flow resistivity (e.g. filter materials).

Measuring the porous properties of a medium is a demanding task. Different parameters are often measured by different application-specific methods, e.g., the porosity of rock is measured by weighing watersaturated samples and comparing their weight with dried samples. The flow resistivity of the damping materials can be computed from the pressure drop caused to the gas flowing through the material. In medical ultrasound studies, the porosity of the bone is determined indirectly by measuring the ultrasound attenuation as the wave passes through the bone.

For the porous material characterization, information carried by waves provides a potential way to estimate the corresponding material parameters. Ultrasound tomography (UST) is one technique that can be used for material characterization purposes. In this technique, an array of sensors is placed around the target. Typically, one of the sensors is acting as a source while others are receiving the data. By changing the sensor that acts as a source, a comprehensive set of wave data can be recorded which can be used to infer the material properties. For further details on the UST, we refer to [13] and references therein. 
The theory of wave propagation in porous media was rigorously formulated in 1950's and 1960's by Biot $[3,4,6,5]$. The model was first used to study the porous properties of bedrock in oil exploration. Since then, the model has been applied and further developed in a number of different fields [32, 35, 21, 31]. The challenge of Biot's model is its computational complexity. The model produces several different types of waveforms, i.e., the fast and slow pressure waves and the shear wave, the computational simulation of which is a demanding task even for modern supercomputers. Computational challenges further increase when attempting to solve inverse problems, as the forward model has to be evaluated several times.

In the work we consider a process for the parameter estimation, comprising two sub-tasks:

Forward model: the simulation of the wave fields for given parameter values. Wave propagation in coupled poroviscoelastic-viscoelastic-acoustic media can be solved using the discontinuous Galerkin (DG) method (see e.g. [18, 11, 34, 14, 12]), which is a well-known numerical approach to numerically solve differential equations. The DG method has properties that makes it well-suited for wave simulations, e.g., the method can be effectively parallelized and it can handle complex geometries and, due to its discontinuous nature, large discontinuities in the material parameters. These are all properties that are essential features for the method to be used in complex wave problems.

Inverse problem: estimation of the parameters from the measurements of wave fields. Neural networks provide a framework to solve the inverse problems: we can train a neural network as a model from wave fields. During last decades, neural networks have been applied in various research areas such as cancer diagnosis [26, 27], forest inventory [29, 30], and groundwater resources [10, 16]. In our problem, the training of a neural network can be carried out using simulated wave fields as the data. Compared to traditional inverse, neural networks has an advantage that it allows computationally efficient inferences. In other words, after the network has been learned, inferences can be carried out using the network without evaluating the forward model. Furthermore, the neural networks provide a straightforward approach to marginalize uninteresting parameters in the inference.

Deep convolutional neural networks (CNN) are a special type of deep neural networks $[25,2,24]$ that employ convolutions instead of matrix multiplications. For example, convolutional layers in the network can be used to make the inference translationally invariant, which is a natural choice for images, see e.g. [20,33]. Furthermore, compared to fully connected networks, convolutional layers also allows significant reduction in the number of parameters in the network. The ultrasound data, considered in this work, can be expressed as images and therefore the convolutional layers can have a potential to solve the inference problems without a large number of unknowns in the network.

The purpose of this paper is to study the feasibility of using a data based machine learning approach in UST to characterize porous material parameters. In the synthetic model setup, we have a cylindrical water tank including an elastic shell layer. Ultrasound sensors are placed inside the water tank. In the model, we place a porous material sample in water and estimate its porosity and tortuosity from the corresponding ultrasound measurements by a convolutional neural network.

The structure of the rest of the paper is as follows. First, in Section 2, we formulate the poroviscoelastic and viscoelastic models. In Section 3, we describe the discontinuous Galerkin method. In Section 4, we provide details of the neural networks technique used in this work and present a numerical experiment. Finally, conclusions are given in Section 5.

\section{GOVERNING EQUATIONS}

In this paper, we consider wave propagation in isotropic coupled poroviscoelastic-viscoelastic-acoustic media. In the following sections, we follow $[8,28,34,12]$ and formulate the poroviscoelastic and viscoelastic wave models. 


\subsection{Biot's poroelastic wave equation}

We use the theory of wave propagation in poroelastic media developed by Biot in $[3,4,6,5]$. We express Biot equations in terms of solid displacement $\mathbf{u}_{\mathrm{s}}$ and the relative displacement of fluid $\mathbf{w}=\phi\left(\mathbf{u}_{\mathrm{f}}-\mathbf{u}_{\mathrm{s}}\right)$, where $\phi$ is the porosity and $\mathbf{u}_{\mathrm{f}}$ is the fluid displacement. In the following, $\rho_{\mathrm{s}}$ and $\rho_{\mathrm{f}}$ denote the solid and fluid densities, respectively. We have

$$
\begin{aligned}
\rho_{\mathrm{a}} \frac{\partial^{2} \mathbf{u}_{\mathrm{s}}}{\partial t^{2}}+\rho_{\mathrm{f}} \frac{\partial^{2} \mathbf{w}}{\partial t^{2}} & =\nabla \cdot \mathbf{T} \\
\rho_{\mathrm{f}} \frac{\partial^{2} \mathbf{u}_{\mathrm{s}}}{\partial t^{2}}+m \frac{\partial^{2} \mathbf{w}}{\partial t^{2}}+\frac{\eta}{k} \frac{\partial \mathbf{w}}{\partial t} & =\nabla \cdot \mathbf{T}_{\mathrm{f}}
\end{aligned}
$$

where $\rho_{\mathrm{a}}$ is the average density

$$
\rho_{\mathrm{a}}=(1-\phi) \rho_{\mathrm{s}}+\phi \rho_{\mathrm{f}}
$$

and the stress $\mathbf{T}=(1-\phi) \mathbf{T}_{\mathrm{s}}+\phi \mathbf{T}_{\mathrm{f}}$, where $\mathbf{T}_{\mathrm{s}}$ is the solid stress and $\mathbf{T}_{\mathrm{f}}$ the fluid stress tensor. Furthermore, in Eq. (2)

$$
m=\rho_{\mathrm{f}} \tau / \phi
$$

where $\tau$ is the tortuosity. In Eq. (2) $\eta$ is the fluid viscosity and $k$ is the permeability. For a detailed discussion we refer to [8].

The third term in (2) is a valid model at low frequencies, when the flow regime is laminar (Poiseuille flow). At high frequencies, inertial forces may dominate the flow regime. In this case the attenuation model may be described in terms of viscous relaxation mechanics as discussed at the end of this Section. A characteristic frequency $f_{c}$ can be used to mark the boundary between the two flow regimes

$$
f_{c}=\frac{\eta \phi}{2 \pi \tau \rho_{\mathrm{f}} k}
$$

In [3] Biot proposed generalized Hooke's laws to describe the stress-strain coupling between the solid and the fluid. Let $\mathbf{E}$ denote the solid strain tensor

$$
\mathbf{E}=\frac{1}{2}\left(\nabla \mathbf{u}_{\mathrm{s}}+\left(\nabla \mathbf{u}_{\mathrm{s}}\right)^{\mathrm{T}}\right)
$$

One can express the solid and fluid stress tensors as

$$
\begin{aligned}
\mathbf{T} & =2 \mu_{\mathrm{fr}} \mathbf{E}+\left(\kappa_{\mathrm{fr}}+\alpha^{2} M-\frac{2}{3} \mu_{\mathrm{fr}}\right) \operatorname{tr}(\mathbf{E}) \mathbf{I}-\alpha M \zeta \mathbf{I} \\
\mathbf{T}_{\mathrm{f}} & =M(\alpha \operatorname{tr}(\mathbf{E})-\zeta) \mathbf{I}
\end{aligned}
$$

where $\mu_{\mathrm{fr}}$ is the frame shear modulus, $\kappa_{\mathrm{fr}}$ is the frame bulk modulus, $\operatorname{tr}(\cdot)$ is the trace, $\mathbf{I}$ is the identity matrix, and $\zeta=-\nabla \cdot \mathbf{w}$ is the variation of the fluid content. In Eqs. (5) and (6), $\alpha$ denotes the Biot effective stress constant given by

$$
\alpha=1-\frac{\kappa_{\mathrm{fr}}}{\kappa_{\mathrm{s}}}
$$

where $\kappa_{\mathrm{s}}$ is the solid modulus. Finally, the modulus $M$ can be written as

$$
M=\frac{\kappa_{\mathrm{s}}}{\alpha-\phi\left(1-\kappa_{\mathrm{s}} / \kappa_{\mathrm{f}}\right)} .
$$

\section{Poroviscoelasticity in the high-frequency regime}

In the high-frequency case, the term $\frac{\eta}{k} \frac{\partial \mathbf{w}}{\partial t}$ in Eq. (2) is replaced by a convolution $b * \frac{\partial^{2} \mathbf{w}}{\partial t^{2}}=b * \frac{\partial \mathbf{v}_{\mathrm{f}}}{\partial t}$, where $\mathbf{v}_{\mathrm{f}}$ denotes the relative fluid velocity and

$$
b=\frac{\eta}{k} \Psi(t) H(t)
$$


In Eq. 8, $\Psi(t)$ is a relaxation function of the form

$$
\Psi(t)=1+\sum_{l=1}^{L_{p}}\left(\frac{\tau_{\epsilon}^{l}}{\tau_{\sigma}^{l}}-1\right) e^{-t / \tau_{\sigma}^{l}},
$$

where $\tau_{\epsilon}$ and $\tau_{\sigma}$ are relaxation times, and $H(t)$ is a Heaviside function. Following [12], we introduce memory variables $\mathbf{e}^{\ell}, \ell=1, \ldots, L_{p}$, leading to an additional differential equations:

$$
\frac{\partial \mathbf{e}^{\ell}}{\partial t}=\left(\frac{\tau_{\epsilon}^{\ell}}{\tau_{\sigma}^{\ell}}-1\right) \frac{\partial \mathbf{v}_{\mathrm{f}}}{\partial t}-\frac{\mathbf{e}^{\ell}}{\tau_{\sigma}^{\ell}}
$$

and

$$
b * \frac{\partial \mathbf{v}_{\mathrm{f}}}{\partial t}=\frac{\eta}{k} \mathbf{v}_{\mathrm{f}}+\frac{\eta}{k} \sum_{\ell=1}^{L_{p}} \mathbf{e}^{\ell} .
$$

In the following, we assume $L_{p}=1$. Relaxation times can be expressed in terms of a quality factor $Q_{0}$ and a reference frequency $f_{0}$ as

$$
\tau_{\epsilon}=\frac{\sqrt{Q_{0}^{2}+1}+1}{2 \pi f_{0} Q_{0}} \text { and } \tau_{\sigma}=\frac{\sqrt{Q_{0}^{2}+1}-1}{2 \pi f_{0} Q_{0}}
$$

\subsection{Viscoelastic wave equation}

The following discussion on the elastic wave equation with viscoelastic effects follows Carcione's book [8], in which a detailed discussion can be found. Expressed as a second order system, the elastic wave equation can be written in the following form

$$
\rho_{\mathrm{e}} \frac{\partial^{2} \mathbf{u}_{\mathrm{e}}}{\partial t^{2}}=\nabla \cdot \mathbf{S}+\mathbf{s}
$$

where $\rho_{\mathrm{e}}$ is the density, $\mathbf{u}_{\mathrm{e}}$ the elastic displacement, $\mathbf{S}$ is a stress tensor, and $\mathbf{s}$ is a volume source. In the two-dimensional viscoelastic (isotropic) case considered here, components of the solid stress tensor $\mathbf{S}$ may be written as $[8]$

$$
\begin{aligned}
\sigma_{11} & =\left(\lambda_{\mathrm{e}}+2 \mu_{\mathrm{e}}\right) \epsilon_{11}+\lambda_{\mathrm{e}} \epsilon_{22}+\left(\lambda_{\mathrm{e}}+\mu_{\mathrm{e}}\right) \sum_{\ell=1}^{L_{e}} \nu_{1}^{(\ell)}+2 \mu_{\mathrm{e}} \sum_{\ell=1}^{L_{e}} \nu_{11}^{(\ell)}, \\
\sigma_{22} & =\left(\lambda_{\mathrm{e}}+2 \mu_{\mathrm{e}}\right) \epsilon_{22}+\lambda_{\mathrm{e}} \epsilon_{11}+\left(\lambda_{\mathrm{e}}+\mu_{\mathrm{e}}\right) \sum_{\ell=1}^{L_{e}} \nu_{1}^{(\ell)}-2 \mu_{\mathrm{e}} \sum_{\ell=1}^{L_{e}} \nu_{11}^{(\ell)}, \\
\sigma_{12} & =2 \mu_{\mathrm{e}} \epsilon_{12}+2 \mu_{\mathrm{e}} \sum_{\ell=1}^{L_{e}} \nu_{12}^{(\ell)},
\end{aligned}
$$

where $\mu_{\mathrm{e}}$ and $\lambda_{\mathrm{e}}$ are the unrelaxed Lamé coefficients, $\epsilon_{11}, \epsilon_{22}$, and $\epsilon_{12}$ are the strain components, and $L_{e}$ is the number of relaxation terms. The memory variables satisfy

$$
\begin{aligned}
\frac{\partial \nu_{1}^{(\ell)}}{\partial t} & =-\frac{\nu_{1}^{(\ell)}}{\tau_{\sigma \ell}^{(1)}}+\phi_{1 \ell}(0)\left(\epsilon_{11}+\epsilon_{22}\right) \\
\frac{\partial \nu_{11}^{(\ell)}}{\partial t} & =-\frac{\nu_{11}^{(\ell)}}{\tau_{\sigma \ell}^{(2)}}+\frac{\phi_{2 \ell}(0)\left(\epsilon_{11}-\epsilon_{22}\right)}{2}, \quad \ell=1, \ldots, L_{e}, \\
\frac{\partial \nu_{12}^{(\ell)}}{\partial t} & =-\frac{\nu_{12}^{(\ell)}}{\tau_{\sigma \ell}^{(2)}}+\phi_{2 \ell}(0) \epsilon_{12},
\end{aligned}
$$


where

$$
\phi_{k \ell}(t)=\frac{1}{\tau_{\sigma \ell}^{(k)}}\left(1-\frac{\tau_{\epsilon \ell}^{(k)}}{\tau_{\sigma \ell}^{(k)}}\right)\left(\sum_{\ell=1}^{n} \frac{\tau_{\epsilon \ell}^{(k)}}{\tau_{\sigma \ell}^{(k)}}\right)^{-1} \exp \left(-t / \tau_{\sigma l}^{(k)}\right), \quad k=1,2
$$

In Eq. $(20) \tau_{\epsilon \ell}^{(k)}$ and $\tau_{\sigma \ell}^{(k)}$ are relaxation times corresponding to dilatational $(k=1)$ and shear $(k=2)$ attenuation mechanisms.

Acoustic wave equation can be obtained from the system by setting the Lamé coefficient $\mu_{\mathrm{e}}$ to zero.

\section{DISCONTINUOUS GALERKIN METHOD}

In the following, we briefly outline the discontinuous Galerkin (DG) method. Our formulation follows [15], where a detailed account of the DG method can be found. We first suppose that the computational domain $\Omega \subset \mathbb{R}^{2}$ is triangulated using $K$ elements

$$
\Omega=\bigcup_{k=1}^{K} D^{k}
$$

The boundary of element $D^{k}$ is denoted by $\partial D^{k}$. We assume that the elements are aligned with material discontinuities. The derivation of the strong form for each element of the computational grid has the following steps:

I. Both of the physical models, poroviscoelastic and viscoelastic wave equations, discussed in Sections 2.1 and 2.2 are first written in the form of a first-order hyperbolic system [12]. This leads one to solve for three strain components $\epsilon_{11}, \epsilon_{22}$, and $\epsilon_{12}$ and four velocity components, $u_{\mathrm{s}}, v_{\mathrm{s}}, u_{\mathrm{f}}$, and $v_{\mathrm{f}}$, variation of the fluid content $\zeta$, and (in the high-frequency regime) two memory variables with the poroviscoelastic model. Similarly, for the viscoelastic case, we have three strain components, two elastic (solid) velocities and, depending on the number of relaxation terms, $3 \times L_{e}$ memory variables.

II. The hyperbolic system discussed in step I is next multiplied with test function and integrated over the volume of the corresponding element $D^{k}$. The integration by parts generates an integral over the element boundary $\partial D^{k}$, which is replaced by the numerical flux. Finally, the system is integrated by parts again.

III. In this paper, we use an upwind numerical flux. The corresponding flux is obtained by solving the exact Riemann problem at the element interfaces [34, 12].

The final strong form is obtained by summing over all elements $K$.

For the hyperbolic system, initial and boundary conditions are needed. In the numerical experiments shown in this paper, initial conditions are always assumed to be zero and we apply the free condition as a boundary condition. For further discussions of boundary conditions, we refer to [8,34] and references therein.

\section{NUMERICAL EXPERIMENT}

In this section, we present the results obtained from testing the data driven approach to estimate porous material parameters in ultrasound tomography.

\subsection{Model setup}

Let us first introduce the model problem. Figure 1 shows the problem geometry. The propagation medium contains three subdomains: a cylindrical shaped poroelastic inclusion (black), a fluid (light gray), and a solid shell (dark gray). The computational domain is an circle with radius of $10 \mathrm{~cm}$ with a $1 \mathrm{~cm}$ thick shell layer. A circular shaped poroelastic inclusion with a radius of $4 \mathrm{~cm}$ is vertically shifted to $2 \mathrm{~cm}$ from the center of 
the circular domain. As already mentioned, the free condition is used along the external boundary. Shifting the target from the center avoids symmetrically positioned sensors (with respect to $\mathrm{x}$-axis) to receive equal signal and therefore have more information from the target.

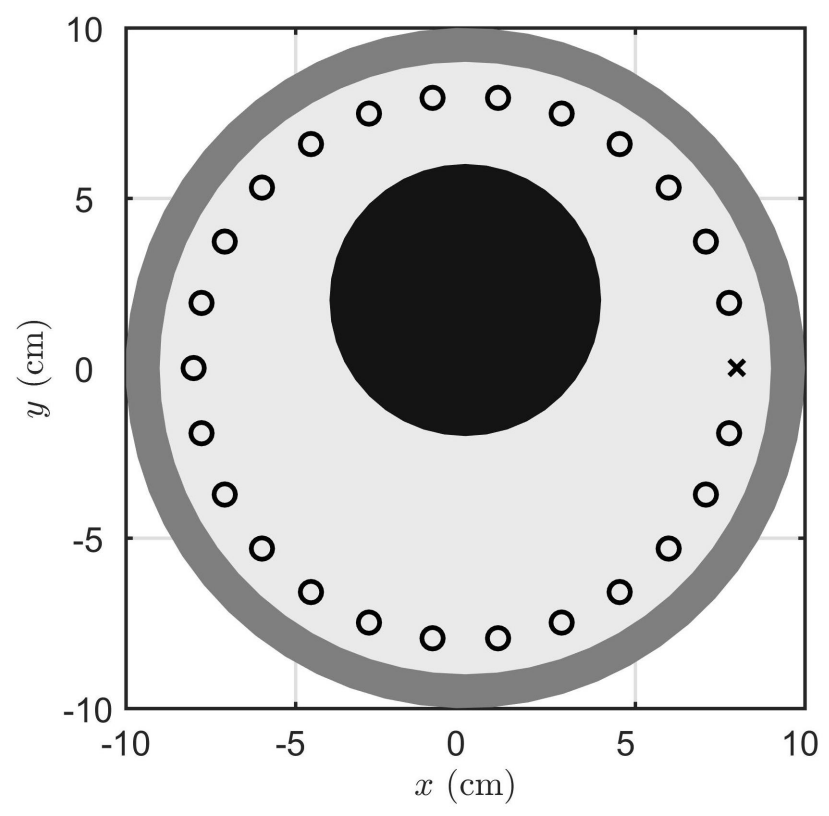

Figure 1: Figure shows the problem geometry. In the graph, the circles denote the receivers and the cross denotes the source.

A total of 26 uniformly distributed ultrasound sensors are located at a distance of $8 \mathrm{~cm}$ from the center of the circle. The source is introduced on the strain components $\epsilon_{11}$ and $\epsilon_{22}$ by the first derivative of a Gaussian function with frequency $f_{0}=40 \mathrm{kHz}$, a time delay $t_{0}=1.2 / f_{0}$. The sensor that is used as a source does not collect data in our simulations. Receivers collect solid velocity components $u_{\mathrm{s}}$ and $v_{\mathrm{s}}$. In the following, the simulation time is $0.4 \mathrm{~ms}$.

Time integration is carried out using an explicit low-storage Runge-Kutta scheme [9]. For each simulation, the length of the time step $\Delta t$ is computed from

$$
\Delta t=\left(\frac{h_{\min }^{\ell}}{2 c_{\max }^{\ell}\left(N^{\ell}\right)^{2}}\right)_{\min }, \quad \ell=1, \ldots, K
$$

where $c_{\max }^{\ell}$ is the maximum wave speed, $N^{\ell}$ is the order of the polynomial basis, $h_{\min }^{\ell}$ is the smallest distance between two vertices in the element $\ell$, and $K$ is the number of elements. Note that recorded data is downsampled to a sampling frequency of $800 \mathrm{kHz}$ on each receiver.

The inclusion is fully saturated with water. The fluid parameters are given by: the density is $\rho_{\mathrm{f}}=1020$ $\mathrm{kg} / \mathrm{m}^{3}$, the fluid bulk modulus is $\kappa_{\mathrm{f}}=2.295 \mathrm{GPa}$, and the viscosity is $\eta=1.0 \mathrm{e}-3 \mathrm{~Pa} \cdot \mathrm{s}$. All other material parameters of the inclusion are assumed to be unknown. In this paper, we assume a relatively wide range of possible parameter combinations, see Table 1. Furthermore, the unknown parameters are assumed to be uncorrelated. The physical parameter space gives $\sim 1.5 \mathrm{kHz}$ as an upper bound for Biot's characteristic frequency $f_{c}$ (see Eq. (4)) and hence we operate in Biot's high-frequency regime $\left(f_{c}<f_{0}\right)$ in all possible parameter combinations.

For the fluid subdomain (water), we set: the density $\rho_{\mathrm{e}}=1020 \mathrm{~kg} / \mathrm{m}^{3}$, the first Lamé parameter $\lambda_{\mathrm{e}}=$ $2.295 \mathrm{GPa}$, and the second Lamé parameter $\mu_{\mathrm{e}}=0$. The elastic shell layer has the following parameters: $\rho_{e}=2000 \mathrm{~kg} / \mathrm{m}^{3}, \lambda_{e}=12.940 \mathrm{GPa}$, and $\mu_{e}=5.78 \mathrm{GPa}$. The relaxation times for the viscoelastic attenuation in the shell layer are given in Table 2 . 
Table 1: Table lists the minimum and maximum values used for uniform distributions for each unknown physical parameter.

\begin{tabular}{cc|cc}
\hline variable name & symbol & minimum & maximum \\
\hline solid density & $\rho_{\mathrm{s}}\left(\mathrm{kg} / \mathrm{m}^{3}\right)$ & 1000.0 & 5000.0 \\
solid bulk modulus & $\kappa_{\mathrm{s}}(\mathrm{GPa})$ & 15.0 & 70.0 \\
frame bulk modulus & $\kappa_{\mathrm{fr}}(\mathrm{GPa})$ & 5.0 & 20.0 \\
frame shear modulus & $\mu_{\mathrm{fr}}(\mathrm{GPa})$ & 3.0 & 14.0 \\
tortuosity & $\tau$ & 1.0 & 4.0 \\
porosity & $\phi$ & 0.01 & 0.99 \\
permeability & $k\left(\mathrm{~m}^{2}\right)$ & $1.0 \mathrm{e}-10$ & $1.0 \mathrm{e}-7$ \\
quality factor & $Q_{0}$ & 20.0 & 150.0 \\
\hline
\end{tabular}

Table 2: Table lists the relaxation times (two mechanisms) used with the shell layer. Relaxation times are computed using the nonlinear optimization method discussed in detail in [7].

\begin{tabular}{c|cccc}
\hline & $\tau_{\epsilon \ell}^{(1)}$ & $\tau_{\sigma \ell}^{(1)}$ & $\tau_{\epsilon \ell}^{(2)}$ & $\tau_{\sigma \ell}^{(2)}$ \\
\hline$\ell=1$ & $1.239 \mathrm{e}-4$ & $1.176 \mathrm{e}-4$ & $1.249 \mathrm{e}-4$ & $1.165 \mathrm{e}-4$ \\
$\ell=2$ & $5.319 \mathrm{e}-6$ & $5.042 \mathrm{e}-6$ & $5.370 \mathrm{e}-6$ & $4.999 \mathrm{e}-6$ \\
\hline
\end{tabular}

The derived wave speeds for each subdomain are given in Table 3. For the poroelastic inclusion, both the minimum and maximum wave speeds are reported. It should be noted that the reported values for the wave speeds in the inclusion correspond to the values generated by sampling the material parameters and hence they may not correspond to the global maximum and minimum values. A detailed approach for calculating wave speeds is given in [12].

Table 3: Derived wave speeds for each subdomain. For the inclusion, both the minimum/maximum values are given. For the inclusion, values are based on sampling the material parameters.

\begin{tabular}{c|ccc}
\hline subdomain & $c_{p}^{1}(\mathrm{~m} / \mathrm{s})$ & $c_{p}^{\mathrm{Il}}(\mathrm{m} / \mathrm{s})$ & $c_{s}(\mathrm{~m} / \mathrm{s})$ \\
\hline inclusion & $1900 / 20189$ & $196 / 1983$ & $833 / 11578$ \\
fluid & 1500 & - & - \\
shell & 3500 & - & 1700 \\
\hline
\end{tabular}

It should be noted that, depending on the application, only some of the material parameters are unknowns of interest. In this work, we focus on estimating the porosity and tortuosity of the inclusion while the solid density and bulk modulus, frame bulk and shear modulus, permeability, and quality factor are marginalized in the neural networks-based inversion algorithm, discussed in detail below.

\subsection{Convolutional neural networks architecture and loss function}

For the convolutional neural networks algorithm used in this work, the recorded wave data is as expressed as images $X \in \mathbb{R}^{d}, d=N_{t} \times N_{r}$, where $N_{t}$ denotes the number of time steps and $N_{r}$ the number of receivers. The input dimension is $d=320 \times 25$, as the data $X$ can be seen as a 2D-image, comprising 25 pixels, corresponding to the receiver positions, times 320 pixels corresponding to the time evolution of the signal.

As the data on each column of the image, we use the following

$$
X(:, \ell)=\frac{x_{r}^{\ell}\left(u_{\mathrm{s}}^{\ell}-u_{\mathrm{s}, n o i}^{\ell}\right)+y_{r}^{\ell}\left(v_{\mathrm{s}}^{\ell}-v_{\mathrm{s}, n o i}^{\ell}\right)}{\sqrt{\left(x_{r}^{\ell}\right)^{2}+\left(y_{r}^{\ell}\right)^{2}}}, \quad \ell=1, \ldots, N_{r},
$$

where $\left(x_{r}^{\ell}, y_{r}^{\ell}\right)$ are the coordinates of the $\ell$ th receiver and $\left(u_{\mathrm{s}}^{\ell}, v_{\mathrm{s}}^{\ell}\right)$ are the horizontal and vertical velocity components on the $\ell$ th receiver. In Eq. (22) subscript noi denotes the data that is simulated without the 
porous inclusion. Two example images are shown in Figure 2. Images show the data for the cases, when the slowest (left) and fastest (right) shear wave speeds are present.
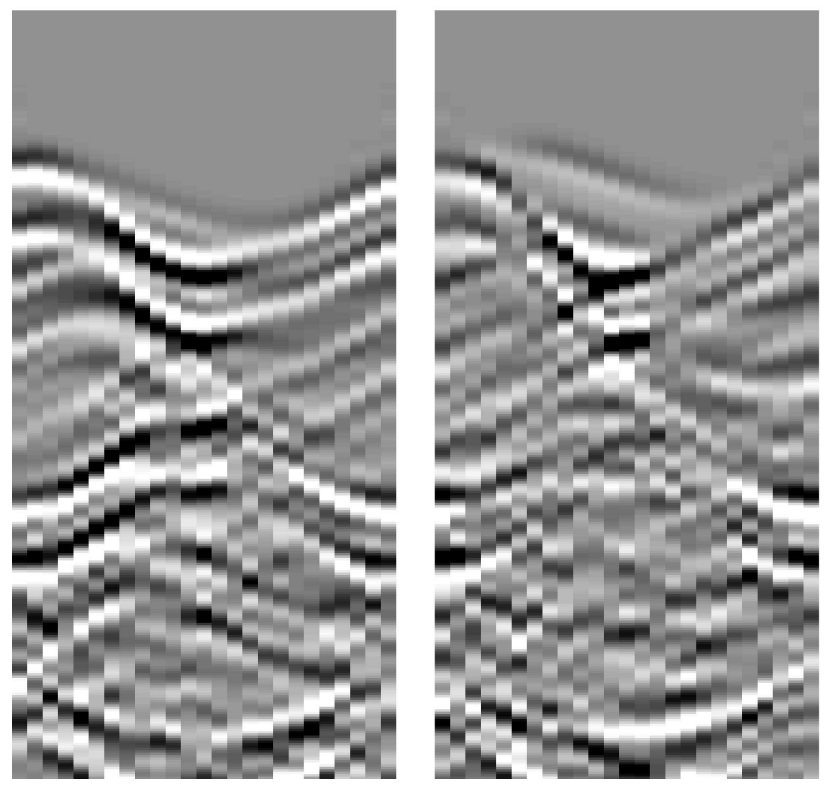

Figure 2: Example images used for training the neural networks algorithm. Note that neural networks algorithm uses the original pixel values of the image $X$ (see Eq. (22)). Time is shown on the vertical-axis and receiver index on the horizontal-axis. Images correspond to samples where the $c_{s}$ is minimum (left) and $c_{s}$ is maximum (right) (see Table 3 ).

A convolutional neural network $\Theta=\Theta(X ; w)$ is a nested composition of functions, from a $d$-dimensional input space $X \in \mathbb{R}^{d}$ to $\Theta \in \mathbb{R}$, which is the output space of the inferred variable. We train a network for both tortuosity and porosity separately. This approach was found to yield superior results, compared to training a network which tries to predict both of the parameters simultaneously. In this work, the network architecture can be expressed as

$$
\Theta(X ; w)=\sigma_{4}\left(w_{4} \cdot \sigma_{3}\left(w_{3} \cdot \sigma_{2}\left(w_{2} * \sigma_{1}\left(w_{1} * X+b_{1}\right)+b_{2}\right)+b_{3}\right)+b_{4}\right),
$$

where $w_{k}$ 's are the parameters of the convolution kernel (filter weights) for the convolutional layers, or the weight matrix for the fully connected layers of the network at level $k$, and $b_{k}$ are bias terms. The functions $\sigma_{k}$ comprises the non-linear ReLU (Rectified Linear Unit), $\sigma=\max (0, X)$ and with the layer normalization[1] and signal dimension reducing pooling layers. The precise model architecture is shown in Table 4.

The training of the neural networks is based on a data set comprising physical parameters $\left\{\Theta_{\ell}\right\}$ and corresponding images $\left\{X_{\ell}\right\}, \ell=1, \ldots, N_{n n}$ (the generation of such a data set is described below). In particular, we are looking at porosity and tortuosity, so $\Theta_{\ell}$ is either $\phi_{\ell}$ or $\tau_{\ell}$. The purpose is to find weights $w$ and biases $b$ that minimize the discrepancy between the physical parameters $\left\{\Theta_{\ell}\right\}$ and the corresponding predicted values given by the neural networks $\left\{\Theta\left(X_{\ell} ; w, b\right)\right\}$. Hence, one is minimizing the quadratic loss function $f\left(w, b ;\left\{X_{\ell}\right\}\right)$ over the simulation dataset

$$
f\left(w, b ;\left\{X_{\ell}\right\}\right)=\frac{1}{N_{n n}} \sum_{\ell=1}^{N_{n n}}\left(\Theta\left(X_{\ell} ; w, b\right)-\Theta_{\ell}\right)^{2}
$$

to obtain the network parameters, weights and biases, of the network. The optimization was carried out with the Adam optimizer[19]. The batch size for the stochastic optimization is chosen to be 50 samples. 
Table 4: The convolutional neural network architecture used in this work. The convolutional layers uses periodic padding in the receiver position-direction. The total number of unknowns in the network is 3.38M.

\begin{tabular}{|c|c|c|c|}
\hline Layer $\mathrm{k}$ & Type and non-linearity & input size & output size \\
\hline & Input & $320 \times 25$ & $320 \times 25 \times 1$ \\
\hline 1 & $\begin{array}{l}\text { Convolution layer }(5 \times 3 \text { filter }) \\
+ \text { Laver normalization }+ \text { ReLU }\end{array}$ & $320 \times 25 \times 1$ & $316 \times 25 \times 20$ \\
\hline & + Max-pooling $(2 \times 2)$ & $316 \times 25 \times 20$ & $158 \times 13 \times 20$ \\
\hline 1 & $\begin{array}{l}\text { Convolution layer }(5 \times 3 \text { filter }) \\
+ \text { Layer normalization }+ \text { ReLU }\end{array}$ & $158 \times 13 \times 20$ & $154 \times 13 \times 25$ \\
\hline 3 & $\begin{array}{c}+ \text { Max-pooling }(2 \times 2) \\
\text { Fully connected }\end{array}$ & $\begin{array}{c}154 \times 13 \times 25 \\
13475\end{array}$ & $77 \times 7 \times 25$ \\
\hline 4 & $\begin{array}{c}+ \text { Layer normalization }+ \text { ReLU } \\
\text { Fully connected layer }\end{array}$ & 250 & $\begin{array}{c}250 \\
1\end{array}$ \\
\hline & Output & & 1 \\
\hline
\end{tabular}

Finally, we to note that purpose aim was not to find the most optimal network architecture. For example, there can be other architectures that can provide similar performance with smaller number of the unknowns. Similar perfomance can also be achieved with fully connected networks with $\sim 3$ layers, but at the expense of significantly larger number of unknowns.

\subsection{Training, validation and test data}

We have generated a training data set comprising 15,000 samples using computational grids that have $\sim 3$ elements per wavelength. The physical parameters for each sample are drawn from the uniform distribution (bounds given in Table 1). The order of the basis functions is selected separately for each element of the grid. The order $N_{\ell}$ of the basis function in element $\ell$ is defined by

$$
N_{\ell}=\left\lceil\frac{2 \pi a h_{\max }^{\ell}}{\lambda_{\ell}^{w}}+b\right\rceil
$$

where $\lambda_{\ell}^{w}=c_{\min }^{\ell} / f_{0}$ is the wavelength, $c_{\min }^{\ell}$ is the minimum wave speed, and $\lceil\cdot\rceil$ is the ceiling function. The parameters $a$ and $b$ control the local accuracy on each element. Following [23, 22], we set $(a, b)=$ $(1.0294,0.7857)$.

Figure 3 shows two examples of computational grids and the corresponding basis order selection on each triangle. Example grids consist of 466 elements and 256 vertices $\left(h_{\min }=0.81 \mathrm{~cm}\right.$ and $\left.h_{\max }=1.95 \mathrm{~cm}\right)$ (sample 1) and 1156 elements and 601 vertices $\left(h_{\min }=0.32 \mathrm{~cm}\right.$ and $\left.h_{\max }=1.75 \mathrm{~cm}\right)($ sample 2$)$.

Figure 4 shows two snapshots of the scattered solid velocity field $\left(\sqrt{\left(u_{\mathrm{s}}-u_{\mathrm{s}, \text { noi }}\right)^{2}+\left(v_{\mathrm{s}}-v_{\mathrm{s}, n o i}\right)^{2}}\right)$ for two time instants. At the first time instant, the transmitted fast pressure wave and also the first reflected wave front is clearly visible. At the second time, all wave components have reflected back from the left surface of the phantom. Furthermore, more complicated wave scattering patterns can be seen inside the porous inclusion. These wave fields demonstrate how the received signals are obtained as combinations of multiple wave fronts.

To include observation noise in the training, each image in the simulation set is copied 5 times and each of the copies is corrupted with Gaussian noise of the form

$$
X_{\ell^{\prime}}^{\text {noised }}=X_{\ell}+A \epsilon^{A}+B\left|X_{\ell}\right| \epsilon^{B}
$$

where $\epsilon^{A}$ and $\epsilon^{B}$ are independent zero-mean identically distributed Gaussian random variables. The second term represents additive white noise and the last term represents noise relative the signal strength. To represent a wide range of different noise levels, for each sample image, the coefficients $A$ and $B$ are randomly 

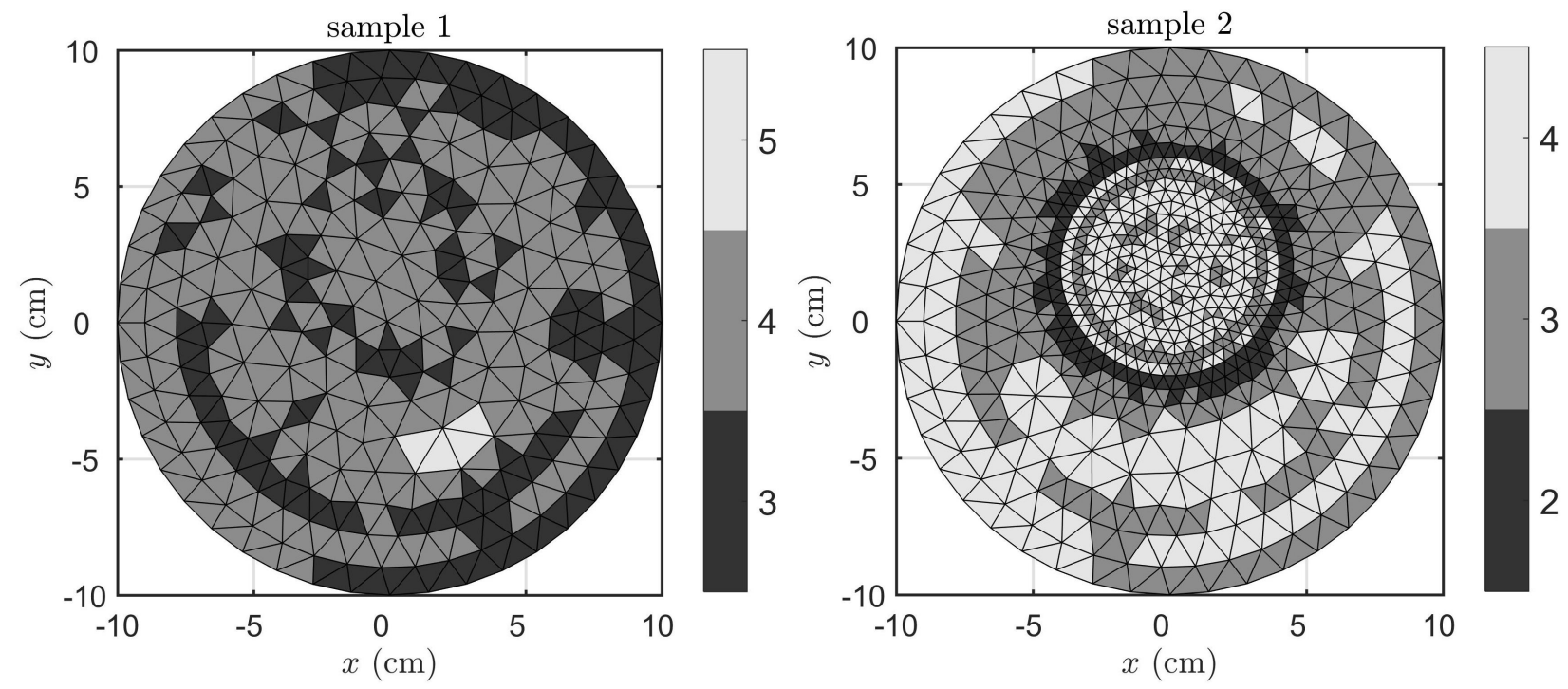

Figure 3: Figure shows two example grids used in the computations. The colorbar shows the order of the basis functions.
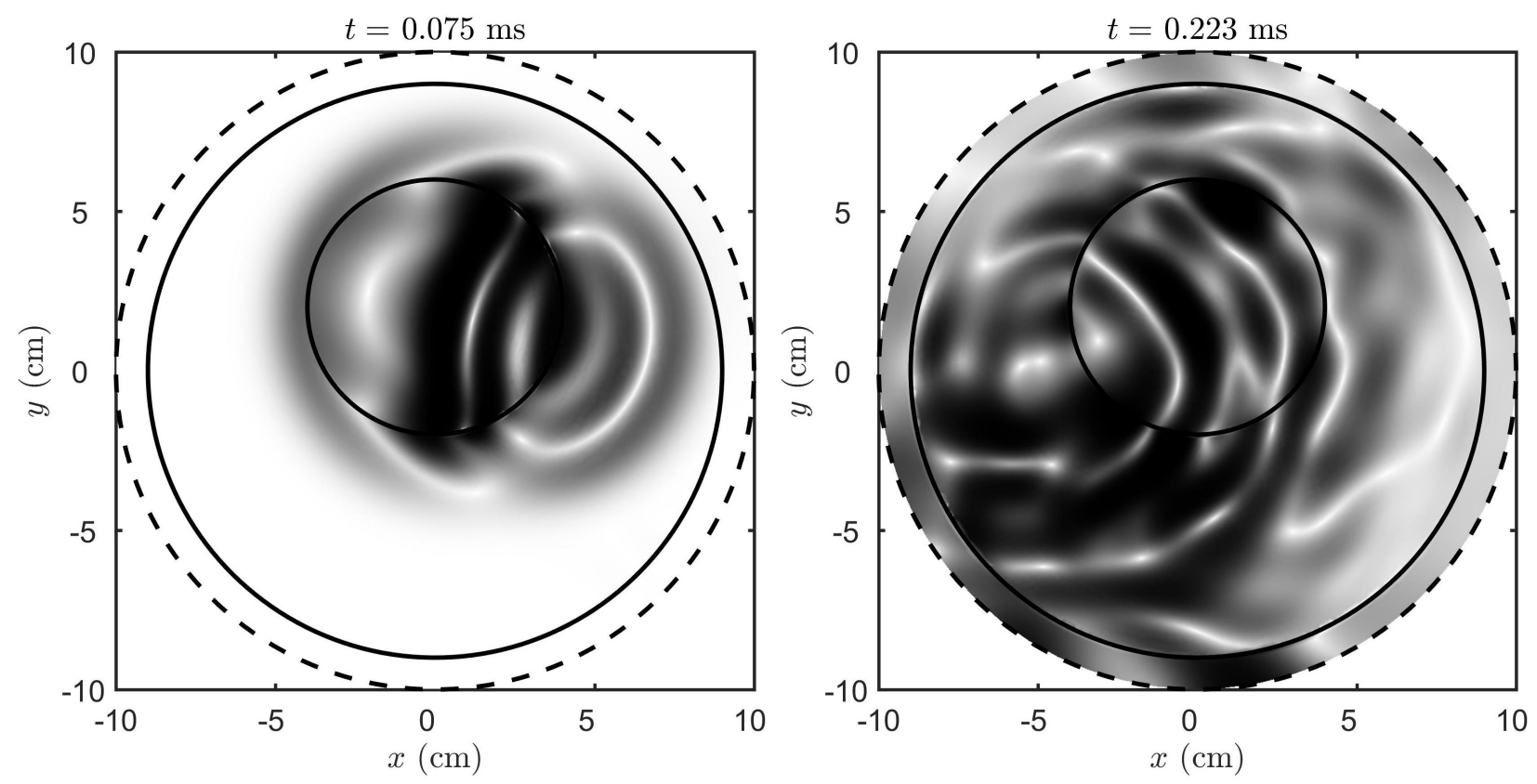

Figure 4: Wave interaction with the poroelastic material at two times. The title shows the time. In the graphs, solid black lines show the inclusion/water and water/shell interfaces while the dotted black line shows the exterior boundary.

chosen such that the standard deviations of the white noise component is between $0.03-5 \%$ (varying logarithmically), and the standard deviations of the relative component is between $0-5 \%$. The total number of samples in the training set is $N_{n n}=5 \times 15000=75000$.

Furthermore, two additional data sets were generated: a validation data set and a test set, that both comprise 3000 samples. In machine learning, the validation data set is traditionally used to evaluate per- 
formance during the training process and the test set is used for the final evaluation of the network. These data sets are generated similarly as the training set, except computational grids were required to have $\sim 4$ elements per wavelength to avoid inverse crime [17]. Furthermore, for the test set, the non-uniform basis order parameters are $(a, b)=(1.2768,1.4384)$ [23] (Eq. (25)) and the noise is added in a more systematic manner (instead of choosing $A$ and $B$ are randomly) to study the performance with different noise levels (see Results section).

\subsection{Results}

Figure 5 shows the loss of the training and validation data. The loss is shown for the two unknown parameters of interest, e.g., porosity and tortuosity. In both cases, we observe that the network has practically reached its generalization capability at least after 2000 full training cycles. The accuracy of the network is affected by the marginalization over all other parameters. In principle, the effect of the other parameters could compensate the changes that using, for example, a different porosity would cause, leaving the waveform of the measurement intact.
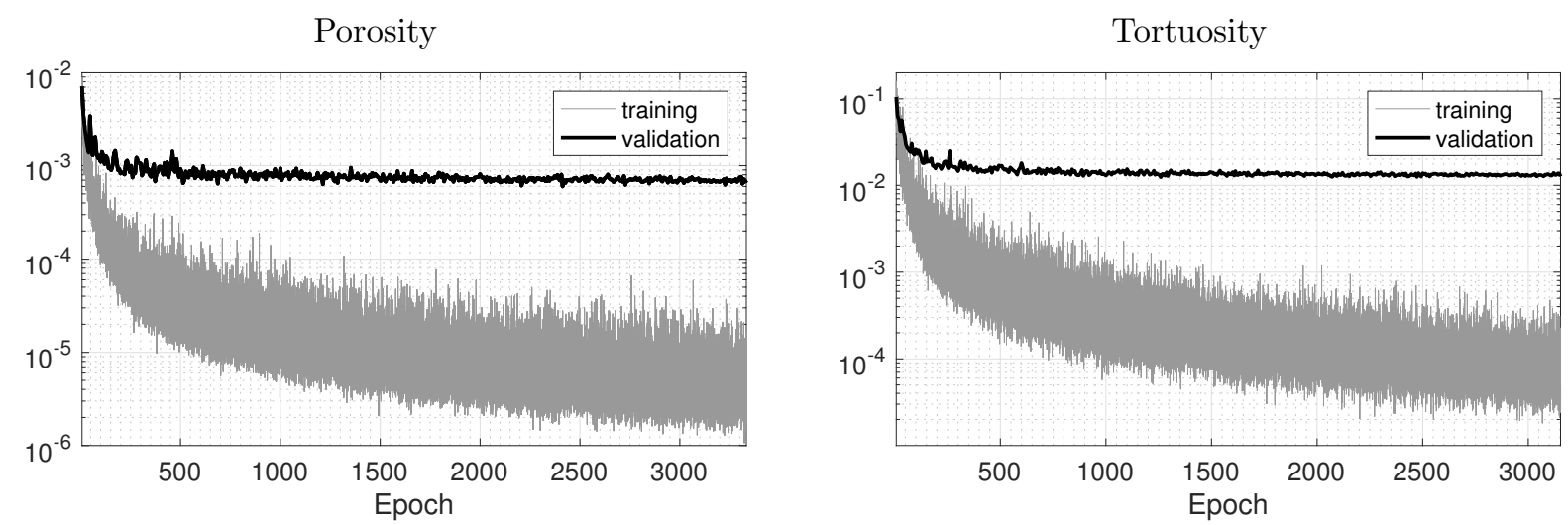

Figure 5: Training and validation loss for porosity (left) and tortuosity (right) as function of number of epochs (full training cycles in stochastic optimization).

We have applied the trained network to predict porosity and tortuosity from images of the test that are corrupted with the white noise component with low noise level (Fig. 6), moderate noise level (Fig. 7) and high noise level (Fig. 8). The figures also include error histograms. Fig. 9 shows the maximum absolute error and the root-mean-square error (the square root of Eq. (24)) as a function of the noise level in white noise component. The predictions are slightly positively biased with smaller noise levels, but positive bias is diminished with higher noise levels. Such a behaviour might be due to the discretization error in forward models (the simulation of training and testing data were carried out using different levels of discretizations) which may dominate with lower observation noise levels but becomes negligible with higer noise levels. On the contrary, with high levels of noise, the predictions are negatively biased especially for larger values of porosity and tortuosity.

We have also studied the effect of the relative noise (the third term in Eq. (26)) to the results. Fig. 10 shows the maximum absolute error and the root-mean-square error (the square root of Eq. (24)) as a function of the noise level in the relative component. As we can see, the predictions are almost uneffected by the relative error even with significantly high noise levels $(\sim 5-10 \%)$.

\section{CONCLUSIONS}

In this paper, we proposed the use of convolutional neural networks (CNN) for estimation of porous material parameters from synthetic ultrasound tomography data. In the studied model, ultrasound data was generated 

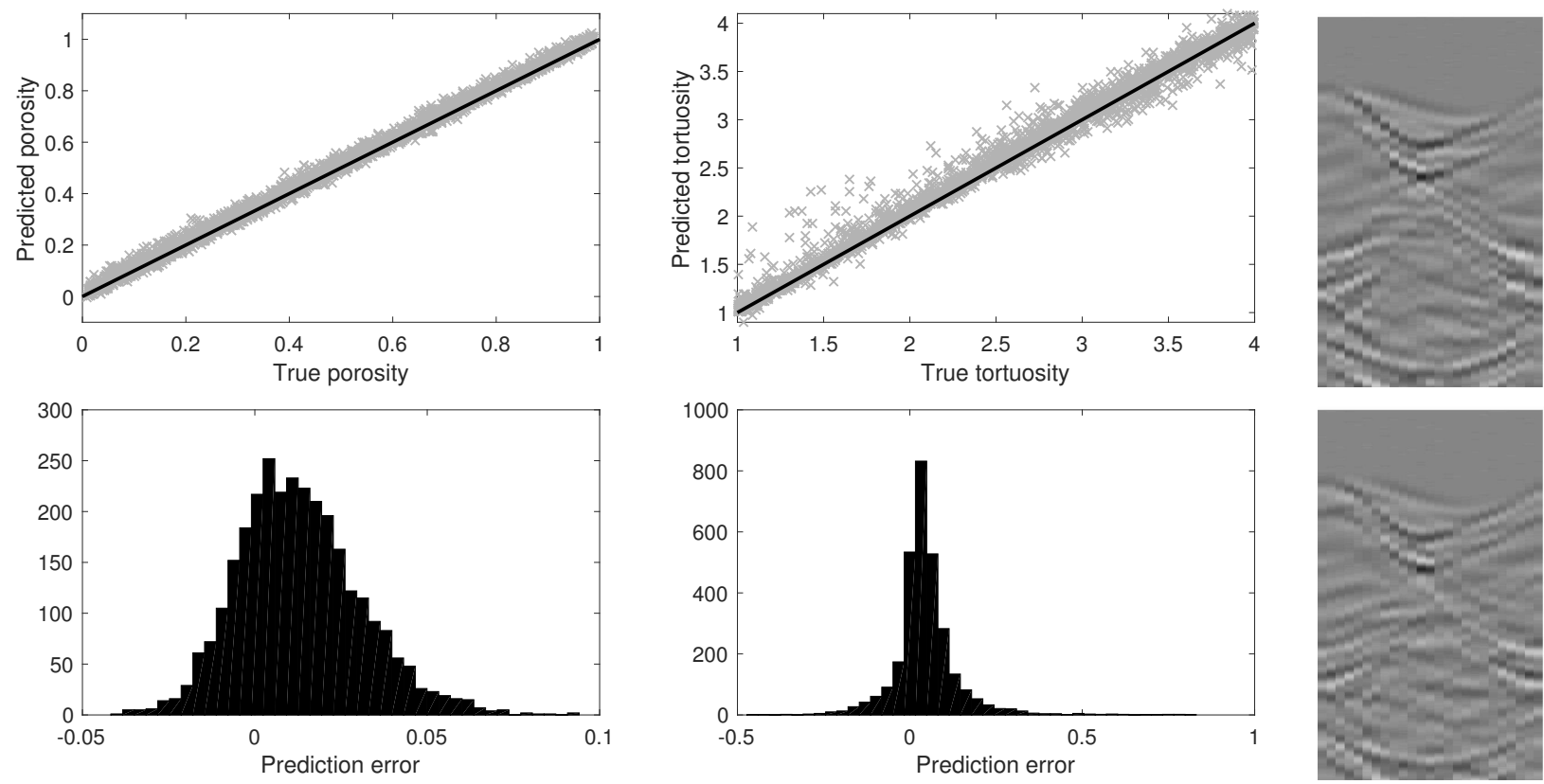

Figure 6: Predicted porosities (left) and tortuosities (middle) for the test data with white noise of $0.8 \%$ (relative to the maximum absolute value of the signal in the training set). Bottom row (left and middle) shows histograms of the prediction error (difference between the predicted and true values). The right column shows two examples of the sample images in this noise level.

in a water tank into which the poroelastic material sample was placed. A total of 26 ultrasound sensors were positioned in the water. One of the sensors generated the source pulse while others were used in the receiving mode.

The recorded velocity data were represented as images which were further used as an input to the CNN. We estimated the porosity and tortuosity of the porous material sample while all other material parameters were marginalized in the solution of the inverse problems.

The reported results support the feasibility of using a data based approach for the characterization of porous material from ultrasound tomography data. Based on the results, it seems that the interesting material parameters can be estimated with acceptable accuracy with wide variety of noise levels, while the uninteresting parameters are successfully marginalized. The success in the marginalization significantly increases the potential of the neural networks for material characterization.

In the experiment, the parameter space for the porous inclusion was assumed to be large. The selected parameter space models a different type of materials and hence the same network can be used to recover the interesting parameters in many different kinds scenarios. For example, the porosity of the inclusion was allowed to span the interwall from $1 \%$ to $99 \%$. The error histograms for the both porosity and tortuosity show excellent accuracy in terms of RMSE and bias. Results also predict that the marginalization of uninteresting parameters can be successfully done by CNN.

The results of this paper support the feasibility of CNN for the characterization of porous materials based on ultrasound tomography data. Future studies should include the extension to three spatial dimensions together with actual measurements.

\section{Acknowledgments}

This work has been supported by the strategic funding of the University of Eastern Finland and by the Academy of Finland (project 250215, Finnish Centre of Excellence in Inverse Problems Research). This 

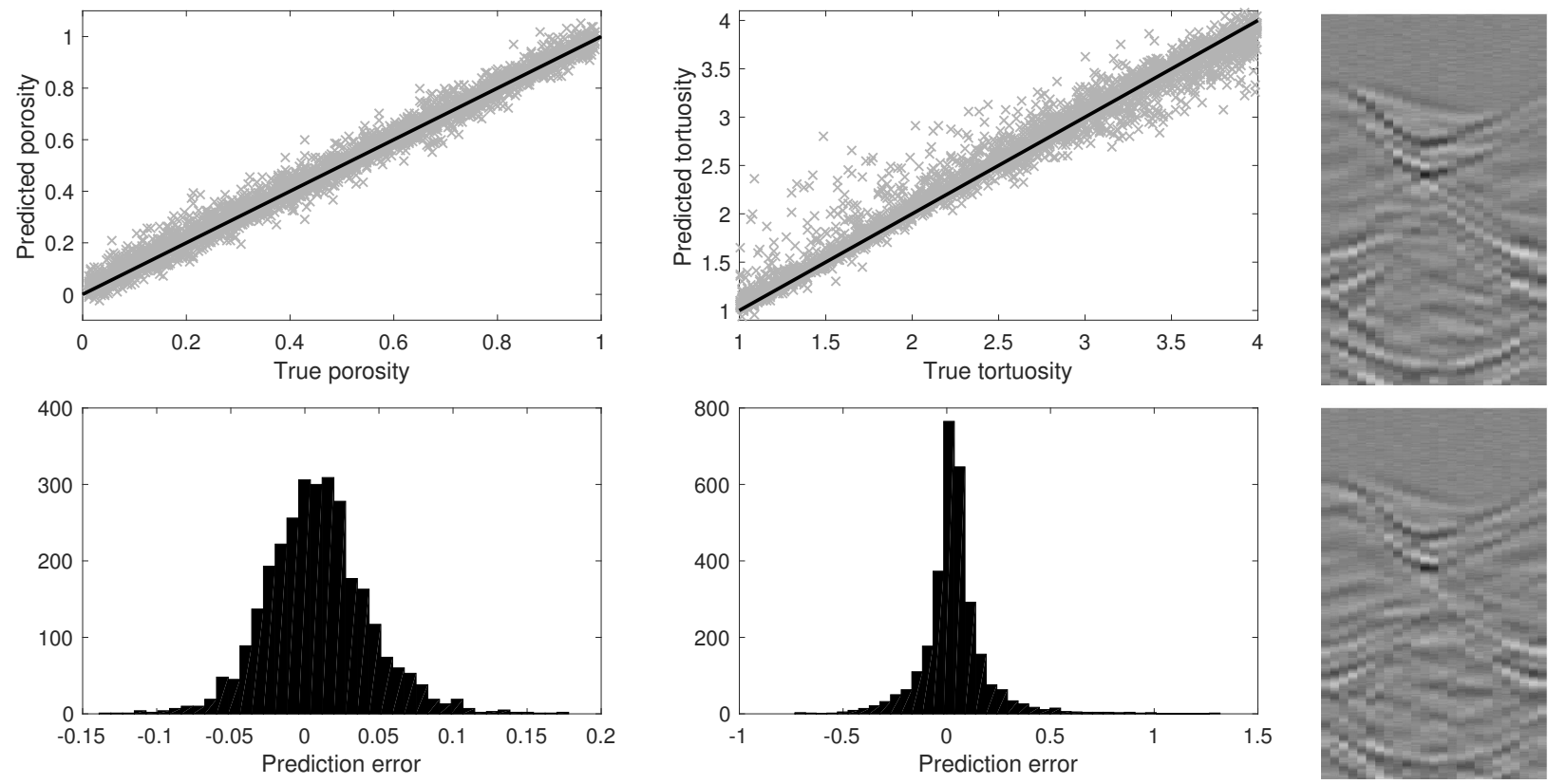

Figure 7: Predicted porosities (left) and tortuosities (middle) for the test data with white noise of $3.2 \%$ (relative to the maximum absolute value of the signal in the training set). Bottom row (left and middle) shows histograms of the prediction error (difference between the predicted and true values). The right column shows two examples of the sample images in this noise level.

article is based upon work from COST Action DENORMS CA-15125, supported by COST (European Cooperation in Science and Technology).

\section{References}

[1] J. Ba, J.R. Kiros, and G.E. Hinton. Layer Normalization. ArXiv e-prints, July 2016.

[2] Y. Bengio. Learning deep architectures for AI. Found. Trends Mach. Learn., 2(1):1-127, 2009.

[3] M.A. Biot. Theory of propagation of elastic waves in a fluid saturated porous solid. I. Low frequency range. J. Acoust. Soc. Am., 28(2):168-178, 1956.

[4] M.A. Biot. Theory of propagation of elastic waves in a fluid saturated porous solid. II. Higher frequency range. J. Acoust. Soc. Am., 28(2):179-191, 1956.

[5] M.A. Biot. Generalized theory of acoustic propagation in porous dissipative media. J. Acoust. Soc. Am., 34(5):1254-1264, 1962.

[6] M.A. Biot. Mechanics of deformation and acoustic propagation in porous media. J. Appl. Phys., 33(4):1482-1498, 1962.

[7] E. Blanc, D. Komatitsch, E. Chaljub, B. Lombard, and Z. Xie. Highly accurate stability-preserving optimization of the Zener viscoelastic model, with application to wave propagation in the presence of strong attenuation. Geophys. J. Int., 205(1):427-439, 2016.

[8] J.M. Carcione. Wave Fields in Real Media: Wave propagation in anisotropic, anelastic and porous media. Elsevier, 2001. 

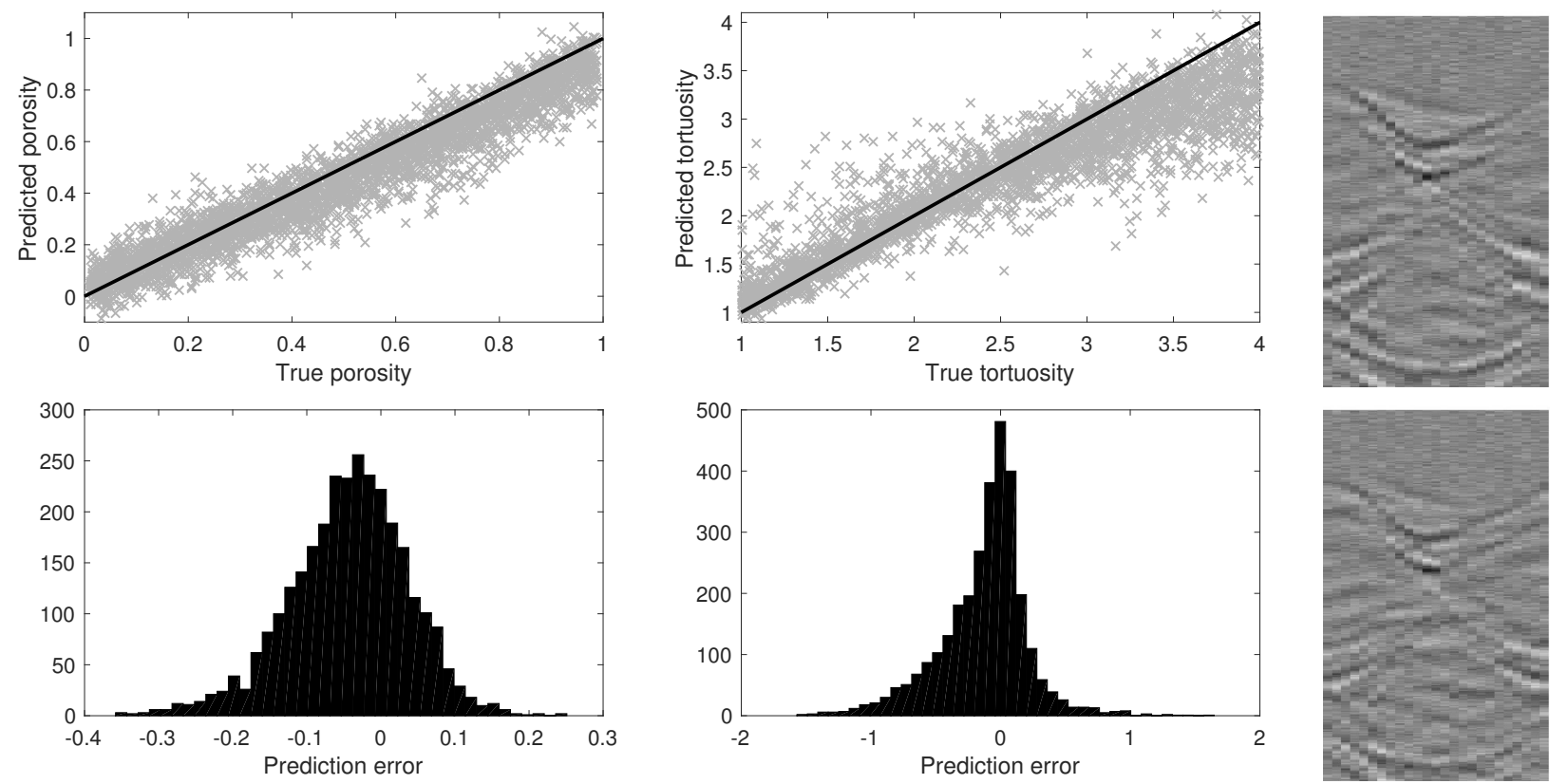

Figure 8: Predicted porosities (left) and tortuosities (middle) for the test data with white noise of $8 \%$ (relative to the maximum absolute value of the signal in the training set). Bottom row (left and middle) shows histograms of the prediction error (difference between the predicted and true values). The right column shows two examples of the sample images in this noise level.

[9] M.H. Carpenter and C.A. Kennedy. Fourth-order 2N-storage Runge-Kutta schemes. Technical report, NASA-TM-109112, 1994.

[10] I. N. Daliakopoulos, P. Coulibaly, and I. K. Tsanis. Groundwater level forecasting using artificial neural networks. J. Hydrol., 309(1-4):229-240, 2005.

[11] J. de la Puente, M. Dumbser, M. Käser, and H. Igel. Discontinuous Galerkin methods for wave propagation in poroelastic media. Geophysics, 73(5):T77-T97, 2008.

[12] N.F. Dudley Ward, T. Lähivaara, and S. Eveson. A discontinuous Galerkin method for poroelastic wave propagation: Two-dimensional case. J. Comput. Phys., 350:690-727, 2017.

[13] N. Duric, P. J. Littrup, C. Li, O. Roy, and S. Schmidt. Ultrasound tomography: A decade-long journey from the laboratory to clinic. In A. Karellas and B. R. Thomadsen, editors, Ultrasound Imaging and Therapy. CRC Press, Taylor \& Francis Group, 2015.

[14] G. Gabard and O. Dazel. A discontinuous Galerkin method with plane waves for sound-absorbing materials. Int. J. Numer. Meth. Eng., 104(12):1115-1138, 2015.

[15] J.S. Hesthaven and T. Warburton. Nodal Discontinuous Galerkin Methods: Algorithms, Analysis, and Applications. Springer, 2007.

[16] M. K. Jha, A. Kumar, and K. P. Sudheer. Artificial neural network modeling for groundwater level forecasting in a River Island of Eastern India. Water Resour. Manage., 24(9):1845-1865, 2010.

[17] J. Kaipio and E. Somersalo. Statistical inverse problems: Discretization, model reduction and inverse crimes. J. Comput. Appl. Math., 198(2):493-504, 2007. 

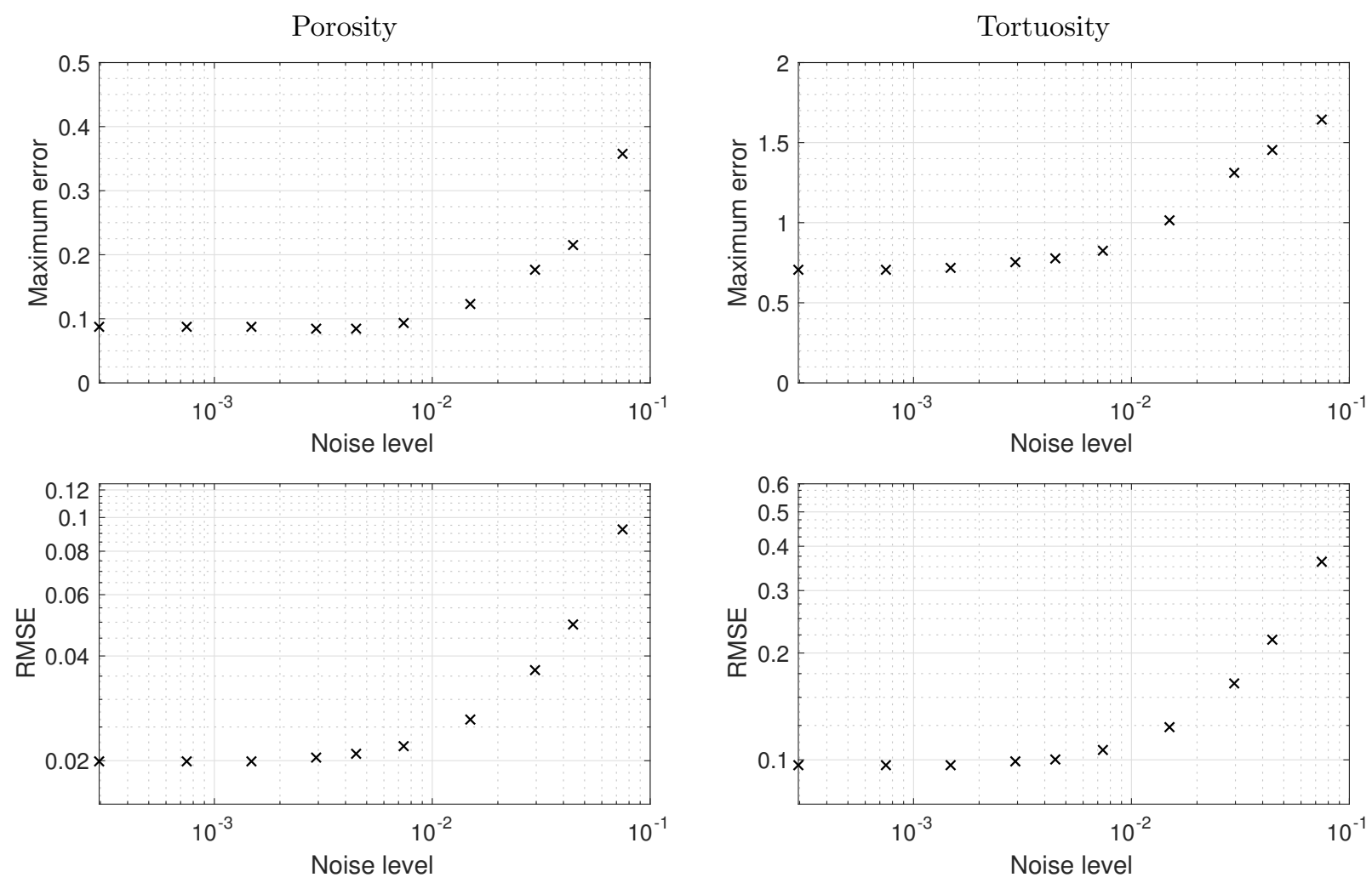

Figure 9: The maximum absolute errors and the root-mean-square errors (RMSE) as a function of the noise level. The $\mathrm{x}$-asis is the noise level relative to the maximum of the (noisefree) signal in the training set.

[18] M. Käser and M. Dumbser. An arbitrary high-order discontinuous Galerkin method for elastic waves on unstructured meshes - I. The two-dimensional isotropic case with external source terms. Geophys. J. Int., 166(23):855-877, 2006.

[19] D.P. Kingma and J. Ba. Adam: A Method for Stochastic Optimization. ArXiv e-prints, December 2014.

[20] A. Krizhevsky, I. Sutskever, and G.E. Hinton. Imagenet classification with deep convolutional neural networks. In F. Pereira, C. J. C. Burges, L. Bottou, and K. Q. Weinberger, editors, Advances in Neural Information Processing Systems 25, pages 1097-1105. Curran Associates, Inc., 2012.

[21] T. Lähivaara, N.F. Dudley Ward, T. Huttunen, Z. Rawlinson, and J.P. Kaipio. Estimation of aquifer dimensions from passive seismic signals in the presence of material and source uncertainties. Geophys. J. Int., 200:1662-1675, 2015.

[22] T. Lähivaara and T. Huttunen. A non-uniform basis order for the discontinuous Galerkin method of the 3D dissipative wave equation with perfectly matched layer. J. Comput. Phys., 229:5144-5160, 2010.

[23] T. Lähivaara and T. Huttunen. A non-uniform basis order for the discontinuous Galerkin method of the acoustic and elastic wave equations. Appl. Numer. Math., 61:473-486, 2011.

[24] Y. LeCun, Y. Bengio, and G. Hinton. Deep learning. Nature, 521(7553):436-444, 2015.

[25] Y. LeCun, L. Bottou, Y. Bengio, and P. Haffner. Gradient-based learning applied to document recognition. Proceedings of the IEEE, 86(11):2278-2324, 1998. 

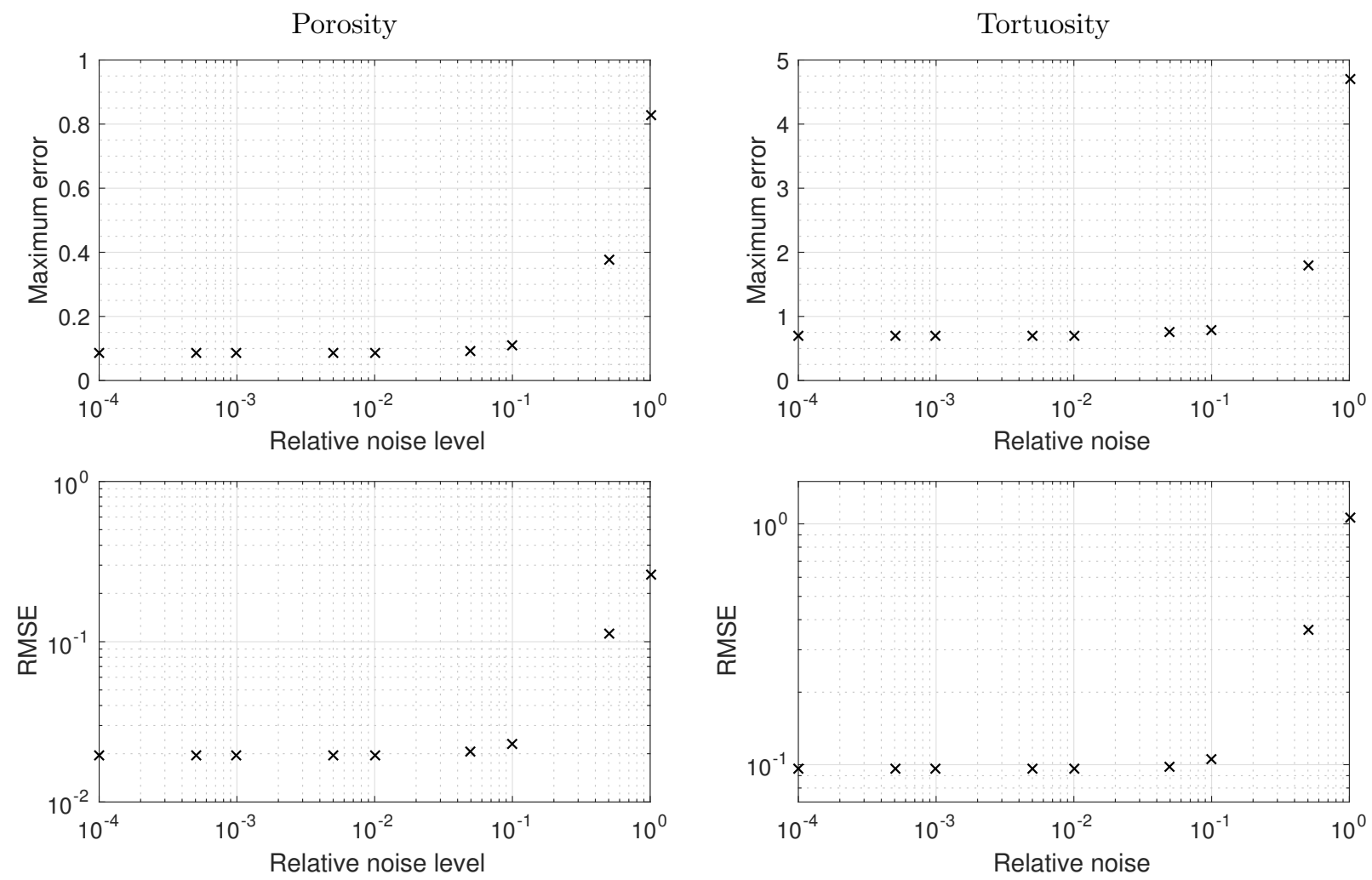

Figure 10: The maximum absolute errors and the root-mean-square errors (RMSE) as a function of the relative noise ( $B$ in Eq. (26)).

[26] P. S. Maclin, J. Dempsey, J. Brooks, and J. Rand. Using neural networks to diagnose cancer. J. Med. Syst., 15(1):11-19, 1991.

[27] L. Alvarez Menendez, F. J. de Cos Juez, F. Sanchez Lasheras, and J.A. Alvarez Riesgo. Artificial neural networks applied to cancer detection in a breast screening programme. Math. Comput. Model., 52(7-8):983-991, 2010.

[28] C. Morency and J. Tromp. Spectral-element simulations of wave propagation in porous media. Geophys. J. Int., 175(1):301-345, 2008.

[29] P. Muukkonen and J. Heiskanen. Estimating biomass for boreal forests using ASTER satellite data combined with standwise forest inventory data. Remote Sens. Environ., 99(4):434-447, 2005.

[30] H. Niska, J-P. Skön, P. Packalen, T. Tokola, M. Maltamo, and M. Kolehmainen. Neural networks for the prediction of species-specific plot volumes using airborne laser scanning and aerial photographs. IEEE Trans. Geosci. Remote Sens., 48(3):1076-1085, 2009.

[31] J. P. Parra Martinez, O. Dazel, P. Göransson, and J. Cuenca. Acoustic analysis of anisotropic poroelastic multilayered systems. J. Appl. Phys., 119(8):084907, 2016.

[32] N. Sebaa, Z.E.A. Fellah, M. Fellah, E. Ogam, A. Wirgin, F.G. Mitri, C. Depollier, and W. Lauriks. Ultrasonic characterization of human cancellous bone using the Biot theory: Inverse problem. J. Acoust. Soc. Am., 120(4):1816-1824, 2006. 
[33] J. Tompson, A. Jain, Y. LeCun, and C.I. Bregler. Joint training of a convolutional network and a graphical model for human pose estimation. In Advances in Neural Information Processing Systems, pages 436-444, 2014.

[34] L.C. Wilcox, G. Stadler, C. Burstedde, and O. Ghattas. A high-order discontinuous Galerkin method for wave propagation through coupled elastic-acoustic media. J. Comput. Phys., 229:9373-9396, 2010.

[35] M. Yvonne Ou. On reconstruction of dynamic permeability and tortuosity from data at distinct frequencies. Inverse Problems, 30(9):095002, 2014. 